\title{
Acute Pancreatitis-A Prospective study of Estimation of Prognosis with MCTSI versus CTSI
}

\author{
Parida $\mathrm{K}^{1}$, Biswal $\mathrm{D}^{2}$ \\ ${ }^{1}$ Dr. Kalyani Parida, Associate Professor, Radiology, affiliated with S.C.B. Medical College \& Hospital, \\ Cuttack, Odisha, India. ${ }^{2}$ Dvijottam Biswal, $3^{\text {rd }}$ year student, affiliated with IMS \& SUM Hospital, Bhubaneswar, \\ Odisha, India.
}

Address for Correspondence: Dr. Kalyani Parida, Email: drkalyaniparida@gmail.com

\begin{abstract}
Introduction: CT is the imaging method of choice for assessing the extent of acute pancreatitis and for evaluating complications. This study was conducted to assess the correlation of modified CT Severity Index (MCTSI) with patient outcome in cases of acute pancreatitis (AP) and to compare it with the CT Severity Index (CTSI). Material and methods: A prospective study of 62 cases was carried out in the Department of Radio Diagnosis, with complaint suggestive of acute pancreatitis on the basis of clinical/laboratory/ultrasonography findings/who were referred for a CECT evaluation of the abdomen and pelvis. The severity of the attack was evaluated using both the indices CT and MCTSI. Correlation between the severity of pancreatitis and the outcome parameters was calculated using the chi square test. Results: When using MCTSI, there was significant correlation between the severity of AP and the outcome parameters such as length of hospital stay $(\mathrm{p}<0.001)$, occurrence of infection $(\mathrm{p}<0.001)$ and organ failure $(\mathrm{p}<0.001)$. CTSI correlated significantly with the occurrence of end organ failure $(\mathrm{p}=0.009)$ and infection $(\mathrm{p}=0.042)$ but less so than MCTSI. In case of intervention, the CTSI $(p<0.001)$ fared slightly better than MCTSI $(\mathrm{p}=0.001)$. There was no significant correlation between the severity of Acute Pancreatitis as assessed by the CTSI and the duration of hospitalization $(\mathrm{p}=0$. 160). Conclusion: The MCTSI correlates significantly with the patient outcome parameters than the CTSI and thus can be useful in predicting the prognosis in patients with acute pancreatitis at an early stage.
\end{abstract}

Keywords- Acute pancreatitis, Modified CT Severity Index (MCTSI), CT Severity Index (CTSI)

\section{Introduction}

Acute Pancreatitis is a very common condition leading to the emergency visits in both developed and developing countries. Computed Tomography plays a pivotal role in the diagnosis and subsequent management of pancreatitis. The modified CT severity index includes a simplified assessment of pancreatic inflammation and necrosis as well as an assessment of extra pancreatic complications [1].

CT is a key diagnostic tool in understanding the cause of endocrine and exocrine pancreatic insufficiency in most patients. Pancreatitis is one of most complex and clinically challenging of all abdominal disorders [2]. The modified Mortele CTSI was easier to calculate and was found to

Manuscript received: $6^{\text {th }}$ May 2017

Reviewed: $16^{\text {th }}$ May 2017

Author Corrected: $24^{\text {th }}$ May 2017

Accepted for Publication: $1^{\text {st }}$ June 2017 correlate more closely with patient outcome measures like the length of the hospital stay, the need for surgery / intervention, and the occurrences of infection, organ failure and death than the currently accepted Balthazar CT severity index, with similar inter observer variability [3]. The revised Atlanta classification system, introduced in 2012, better defined the clinical diagnosis, Computed Tomographic (CT) manifestations and disease course of acute pancreatitis into two morphologic subtypes [4]. Interstitial oedematous pancreatitis and Necrotizing pancreatitis. Modified CT severity index makes the score easier to calculate and reduces the inter-observer variation. Scores obtained with the modified Mortele index, show a stronger statistical correlation for all clinical outcome parameters in all the patients better than the Balthazar index [5]. 


\section{Material and Methods}

This study was conducted in the Department of Radio-Diagnosis, S.C.B. Medical College, Cuttack. The study comprised of 62 patients with a clinical suspicion of Acute Pancreatitis who were referred for a CECT evaluation of the abdomen and pelvis.

Study Design: Prospective study

Inclusion Criteria: Patients with clinically suspected acute pancreatitis who underwent MDCT with contrast within three days of admission.

Exclusion Criteria: Patients with clinically suspected acute pancreatitis who did not undergo MDCT with contrast and Acute pancreatitis due to trauma.

Collection of data- The study was a prospective analysis of CTs of patients who were admitted at our institution with a diagnosis of acute pancreatitis. The protocol was approved by the institute ethics committee. We included patients with complaint suggestive of acute pancreatitis on the basis of clinical/ laboratory/ ultrasonography findings / who were referred for a CECT evaluation of the abdomen and pelvis.

Methods- The patient was explained prior to the procedure and written consent was taken from the patient.The patient was asked to be in overnight nil - oral status and after obtaining renal function tests the contrast enhanced CT was done.

Equipment used - GE Bright speed 16 - slice Multi Detector Computed Tomography Scanner. Plain and post contrast series of the abdomen and pelvis were taken. Acquisition of contiguous axial sections of thickness $5 \mathrm{~mm}$ of abdomen and pelvis was done in a cranio - caudal direction from the level of the xiphisternum to pubic symphysis before and after administration of oral and intravenous non ionic iodinated contrast of $80-$ $100 \mathrm{ml}$. Images were reconstructed with $1.25 \mathrm{~mm}$ slice thickness and reformatted in sagittal and coronal planes for analysis.

The severity is classified into three categories based on clinical and morphologic findings according to revised Atlanta classification [6]

1. Mild - No organ failure and no local or systemic complications.

2. Moderate - Presence of transient organ failure less than $48 \mathrm{~h}$ and/or presence of local complications.

3. Severe - Persistent organ failure $>48$ hour.

The severity of the acute pancreatitis was scored using both the CT Severity Index and the modified CT Severity Index and the patients were followed up prospectively for evaluation of the clinical outcome. The outcome parameters included the length of hospital stay, the need for intervention and the occurrence of infection and organ failure. The clinical outcome was compared with the currently accepted Balthazar's CTSI and Modified Mortele's CTSI in all the cases.

Statistical Analysis- Data analysis was done using SPSS version 16.0 Data transformation by recoding, counting and cross tabulation was performed and obtained information was processed using Pearson chi-square and Fisher's-exact test.

\section{Results}

The study was conducted in the Department of Radio-diagnosis, S.C.B. Medical College, Cuttack during which period 62 patients with a clinical suspicion of Acute pancreatitis underwent a contrast enhanced CT examination within 3 days of hospital admission.

Table-1: Age distribution of the study group.

\begin{tabular}{|c|c|c|}
\hline Age group (in years) & No. of patients & Percentage (\%) \\
\hline$\leq 25$ & 07 & 11 \\
\hline $26-35$ & 25 & 40 \\
\hline $36-45$ & 12 & 19 \\
\hline $46-55$ & 09 & 15 \\
\hline$>55$ & 09 & $\mathbf{1 0 0}$ \\
\hline Total & $\mathbf{6 2}$ & \\
\hline
\end{tabular}


The study included patients between the age group of 10 and 80 years with a mean age of 39 yrs. Maximum number of patients was seen in the 26 - 35 yrs age group which comprised of 25 (40\%) patients.

Table-2: Sex distribution of the study group.

\begin{tabular}{|c|c|c|}
\hline Sex & No. of patients & Percentage (\%) \\
\hline MALE & 47 & 76 \\
\hline FEMALE & 15 & 24 \\
\hline Total & $\mathbf{6 2}$ & $\mathbf{1 0 0}$ \\
\hline
\end{tabular}

In our study, out of 62 cases,47(76\%) were males and 15(24\%) were females with a male : female ratio of 3.1:1.

Table-3: Grading of Severity of acute pancreatitis according to MCTSI.

\begin{tabular}{|c|c|c|}
\hline MCTSI & No. of patients & Percentage \\
\hline MILD(0-2) & 24 & 39 \\
\hline MODERATE (4-6) & 27 & 43 \\
\hline SEVERE(8-10) & 11 & 18 \\
\hline
\end{tabular}

According to the MCTSI, 24 (39\%) patients had mild, 27 (43\%) had moderate and $11(18 \%)$ patients had severe pancreatitis.

Table-4: Grading of severity of AP according to CTSI.

\begin{tabular}{|c|c|c|}
\hline CTSI & No. of patients & Percentage \\
\hline MILD $(0-3)$ & 45 & 72 \\
\hline MODERATE(4-6) & 16 & 26 \\
\hline SEVERE(7-10) & 01 & 02 \\
\hline
\end{tabular}

According to CTSI, 45 (72\%) patients had mild, 16 (26\%) had moderate and 1 (2\%) had severe pancreatitis.

Table 5: Occurrence of organ failure in relation to severity of AP according to MCTSI.

\begin{tabular}{|c|c|c|}
\hline MCTSI & No. of patients & Percentage \\
\hline MILD & 07 & 29 \\
\hline MODERATE & 10 & 100 \\
\hline SEVERE & 11 & 37 \\
\hline
\end{tabular}

Out of 62 patients, 28 (45\%) patients developed organ failure. 18 patients had hepatic failure, 4 cardiac failure, 3 renal failure, 2 respiratory failure, $1 \mathrm{CNS}$ failure and 1 patient had hematologic system failure.

According to MCTSI, of the 28 patients who developed organ failure, 7 patients had mild, 10 had moderate and 11 patients had severe AP. $29 \%$ of patients who had mild pancreatitis had organ failure whereas organ failure was seen in $37 \%$ and $100 \%$ of patients who had moderate and severe AP respectively ( $\mathrm{p}$ value $<0.001$ ).

Table-6: Occurrence of organ failure in relation to severity of AP according to CTSI

\begin{tabular}{|c|c|c|}
\hline CTSI & No. of patients & Percentage \\
\hline MILD & 15 & 33 \\
\hline MODERATE & 12 & 75 \\
\hline SEVERE & 01 & 100 \\
\hline
\end{tabular}

According to CTSI, of these 28 patients, 15 patients had mild, 12 had moderate and 1 patient had severe AP. $33 \%$ of patients who had mild AP had organ failure whereas organ failure was seen in $75 \%$ and $100 \%$ of patients who had moderate and severe AP respectively ( $\mathrm{p}$ value $=0.009$ ). 
The above statistics show that although both the indices show significant correlation with organ failure, highly significant correlation exists between the organ failure with the classification according to MCTSI (p value $<$ 0.001 ) than CTSI ( $\mathrm{p}$ value $=0.009$ ).

Table-7 -Occurrence of infection in relation to severity of AP according to MCTSI.

\begin{tabular}{|c|c|c|}
\hline MCTSI & No. of patients & Percentage \\
\hline MILD & 02 & 08 \\
\hline MODERATE & 10 & 37 \\
\hline SEVERE & 10 & 91 \\
\hline
\end{tabular}

$22(36 \%)$ patients developed systemic infection.According to MCTSI, of these 22 patients, 2 had mild, 10 had moderate and 10 had severe AP. $8 \%$ of mild AP , 37\% of moderate AP and 91\% of severe AP showed evidence of infection ( $p$ value $<0.001$ ).

Table-8: Occurrence of infection in relation to severity of AP according to CTSI.

\begin{tabular}{|c|c|c|}
\hline CTSI & No. of patients & Percentage \\
\hline MILD & 12 & 27 \\
\hline MODERATE & 09 & 56 \\
\hline SEVERE & 01 & 100 \\
\hline
\end{tabular}

According to CTSI, out of 22 patients who developed infection, 12 had mild, 9 had moderate and 1 had severe AP. $27 \%$ of mild AP, $56 \%$ of moderate AP and $100 \%$ of severe AP had infection ( $p$ value $=0.042$ ).

The grade of severity of AP as predicted by MCTSI shows highly significant correlation with the occurrence of infection ( $p$ value $<0.001$ ). The classification according to CTSI also shows significant correlation with the development of infection ( $p$ value $=0.042$ ) but less so than that of MCTSI.

Table-9: Mean duration of hospitalization in relation to severity of AP according to MCTSI.

\begin{tabular}{|c|c|}
\hline MCTSI & Mean duration of hospitalization \\
\hline MILD & 05 \\
\hline MODERATE & 09 \\
\hline SEVERE & 16 \\
\hline
\end{tabular}

Mean duration of hospitalization was 5 days for mild AP, 9 days for moderate and 16 days for severe AP according to the classification by MCTSI ( $p$ value $<0.001$ ).

Table-10: Mean duration of hospitalization in relation to severity of AP according to CTSI.

\begin{tabular}{|c|c|}
\hline CTSI & Mean duration of hospitalization \\
\hline Mild & 07 \\
\hline Moderate & 13 \\
\hline Severe & 14 \\
\hline
\end{tabular}

According to CTSI, mean duration of hospitalization was 7 days for mild, 13 days for moderate and 14 days for severe AP ( $\mathrm{p}$ value $=0.160)$.

\section{Discussion}

Mean age of presentation is 39 years in our study group and the maximum no. of cases of AP are in the 26 - 35 yr age group. According to Baig et al [7] who conducted a prospective study of the aetiology, severity and outcome of acute pancreatitis in Eastern India, mean age was $30 \mathrm{yrs}$. In studies by Mortele et al [8] and Bollen et al [9] mean age was 49 and 53 yrs respectively. 
In our study, $76 \%$ are males and $24 \%$ are females with a male to female ratio of 3.1:1. Similar results were seen by Baig et al [7] in whose study male to female ratio is $2.75: 1$ with $73 \%$ males and $27 \%$ females. In our study, both the CTSI and the MCTSI show significant correlation with organ failure but highly significant correlation exists between the severity of AP and organ failure with the classification according to MCTSI ( $p$ value < 0.001) than CTSI ( $\mathrm{p}$ value $=0.009$ )

According to Mortele et al [8], a significant correlation $(p=0.0024)$ was seen between the MCTSI and the development of organ failure, but the CTSI did not correlate significantly with the development of organ failure $(\mathrm{p}=0.0513)$.

In our study, the grade of severity of AP as predicted by MCTSI shows highly significant correlation with the occurrence of infection ( $p$ value $<0.001)$. The classification according to CTSI also shows significant correlation with the development of infection ( $p$ value $=0.042$ ) but less so than that of MCTSI. According to our study, the grade of severity of AP as predicted by MCTSI shows significant correlation with the mean duration of hospitalization ( $p$ value $<0.001$ ). On the other hand, the classification according to CTSI does not show significant correlation with the same $(\mathrm{p}$ value $=0.160)$.

Similar results were seen by Mortele et al [8] who found a statistically significant correlation between the MCTSI and the length of hospital stay ( $\mathrm{p}=$ 0.035 ) for all severity groups.However, when using the CTSI, no significant difference $(p=0.15)$ in the length of the hospital stay was seen between the moderate and severe pancreatitis group.

In our study, both the indices correlated significantly with intervention but CTSI had a better correlation ( $p$ value $<0.001$ ) than MCTSI ( $p$ value $=0.001)$. According to Bollen et al [9], for both CT indices, a significant relationship was observed between the score obtained and the severity parameters studied $(\mathrm{p}<0.0001)$. The MCTSI had the higher AUC for the severity parameters of death and ICU stay, whereas the CTSI had higher AUCs for persistent organ failure, need for intervention, and pancreatic infection. However, for all severity parameters studied, no statistically significant difference was observed between the CTSI and MCTSI. In our study mortality rate was $2 \%$. Similar mortality rate was seen by Mortele et al (2\%) [8]. In the study by Bollen et al [9] mortality rate was 6\%. According to D.Yadav and A. B. Lowenfels [10], AP has an overall low mortality, of approximately $1 \%$ and the risk of death increases with age, co-morbidities, and severe disease.

Similar trends in duration of hospital stay, intervention or surgery, evidence of infection, organ failure, and mortality in patients with variable grades of severity of pancreatitis were observed in our study as that seen by Mortele in their study. This also correlated with the study by Irshad Ahmad Banday et al[11], which concluded that Modified CT Severity Index is a simpler scoring tool and more accurate than the Balthazar CT Severity Index [11].

Results of our study were also found similar to a study conducted by ShivanandMelkundi et al., which showed a significant correlation of grades of severity of acute pancreatitis based on MCTSI with patient outcome parameters than grades of severity of acute pancreatitis based on CTSI [12].

Patient outcome in terms of organ failure and death is more accurately assessed by revised Atlanta classification in comparison with Balthazar and modified ct severity index. The revised classification seems to be a good predictor for clinical outcome of AP Shyu JY et al [13].

This CT Severity Index was found to have a better prognostic accuracy than the earlier score but it, too, was found to have some limitations. First, the score obtained with the index did not incorporate the presence of organ failure [14] extra pancreatic parenchymal complications [15] or peripancreatic vascular complications [16] and their correlation with the final outcome. Secondly, as documented in some studies, inter-observer agreement for scoring the CT scans using the CT Severity Index was only moderate, with a reported agreement of approximately $75 \%[17,18]$.

The source of this variability possibly relates to the subjective and multiple categorization of the extent of pancreatic inflammation and necrosis. Vishal Sharma et al [19] found that the prognostic performance of various clinical and radiological 
scoring systems in AP is comparable with BISAP having the highest accuracy for predicting POF and mortality.

ChenyangChen[20] found that Patients $(n=208)$ who underwent abdominal computed tomography (CT) within 24hours after AP onset and admission were retrospectively identified. Each patient's EPIC score, Balthazar score, bedside index of severity in acute pancreatitis (BISAP), and systemic inflammatory response syndrome (SIRS) score were obtained. Primary endpoints were organ failure occurrence and death. Scores were evaluated by receiver operator characteristic (ROC) curve and area under the curve (AUC) analysis.

This difference in statistical significance between CTSI and MCTSI in our study may be attributed to the inclusion of extrapancreatic complications in the MCTSI system. We assume that the presence of ascites and pleural fluid may be responsible for the improved correlation with MCTSI, because they may be early indicators of organ dysfunction. Another important difference between the MCTSI and CTSI is that, MCTSI differentiates only between presence and absence of acute fluid collections and, therefore does not require a count of the collections as in case of CTSI.

\section{Conclusion}

When using MCTSI, there was highly significant correlation between the severity of Acute Pancreatitis and the outcome parameters such as length of hospital stay, need for intervention, occurrence of infection and organ failure than the CTSI and thus can be useful in predicting the prognosis in patients with acute pancreatitis at an early stage.

Limitation of study is repeated follow-up study was not possible due to cost and radiation exposure. Different treatments were given to patients which changed the patient outcome. Sample size was small which may have affected the result. As per the revised Atlanta classification, CECT is useful after first week. However, in first week only clinical parameters are useful. So future studies are required with Large sample study for accurate results.

\section{Funding: Nil, Conflict of interest: None Permission of IRB: Yes}

\section{Bibliography}

1. Banday IA, Gattoo I, Khan AM, Javeed J, Gupta G, Latief M. Modified Computed Tomography Severity Index for Evaluation of Acute Pancreatitis and its Correlation with Clinical Outcome: A Tertiary Care Hospital Based Observational Study. J Clin Diagn Res. 2015 Aug;9(8):TC01-5. doi: 10. 7860/JCDR/2015/14824.6368. Epub 2015 Aug 1.

2. Manfredi R, Brizi MG, Canadè A, Vecchioli A, Marano P. Imaging of acute pancreatitis. Rays. 2001 Apr-Jun;26(2):135-42.

3. Mortelé KJ, Mergo PJ, Taylor HM, Wiesner W, Cantisani V, Ernst MD, Kalantari BN, Ros PR. Peripancreatic vascular abnormalities complicating acute pancreatitis: contrast-enhanced helical CT findings. Eur J Radiol. 2004 Oct;52(1):67-72.

4. Han J, Kim HG, Jung MK, Cho CM, Cho K, $\mathrm{Kim} \mathrm{KH}$, et al. Revised Atlanta classification of acute pancreatitis can predict clinical outcome better: a retrospective, multicenter study. Pancreatology. 2014;14(3):S32.

5. Raghuwanshi S,Gupta R.CT Evaluation of Acute Pancreatitis and its Prognostic Correlation with CT Severity Index J ClinDiagn Res. 2016 Jun; 10(6): TC06-TC11. Published online 2016 Jun 1.

6. Banks PA, Bollen TL, Dervenis C, Gooszen HG, Johnson CD, Sarr MG, Tsiotos GG, Vege SS; Acute Pancreatitis Classification Working Group. Classification of acute pancreatitis--2012: revision of the Atlanta classification and definitions by international consensus. Gut.2013Jan;62(1):102-11. doi:10.1136/gutjnl-2012-302779.Epub2012 Oct 25.

7. Baig SJ, Rahed A, Sen S.A prospective study of the aetiology, severity and outcome of acute pancreatitis in Eastern India.TropGastroenterol. 2008 Jan-Mar;29(1):20-22.

8. Mortele $\mathrm{KJ}$, Wiesner $\mathrm{W}$, Intriere $\mathrm{L}$, et al: A Modified CT Severity Index for evaluating acute pancreatitis: Improved correlation with patient outcome. AJR 2004;183:1261-1265.

9. Bollen TL, Singh VK, Maurer R, Repas K, van Es HW, Banks PA, Mortele KJ. Comparative evaluation of the modified CT severity index and CT severity index in assessing severity of acute 
pancreatitis. AJR Am J Roentgenol. 2011 Aug;197 (2): 386-92. doi: 10.2214/AJR.09.4025.

10. Yadav D, Lowenfels AB. The epidemiology of pancreatitis and pancreatic cancer. Gastroenterology. 2013 Jun;144(6):1252-61. doi: 10. $1053 /$ j. gastro. 2013.01.068.

11. Banday IA, Gattoo I, Khan AM, Javeed J, Gupta G, Latief M.Modified Computed Tomography Severity Index for Evaluation of Acute Pancreatitis and its Correlation with Clinical Outcome: A Tertiary Care Hospital Based Observational Study. J ClinDiagn Res. 2015 Aug; 9 (8): TC01-5.

12. Melkundi S, Anand N. Modified computed tomography severity index in acute pancreatitis. Journal of Evolution of Medical and Dental Sciences. 2014;3(74):15541-51.h

13. Shyu JY, Sainani NI, Sahni VA, Chick JF, Chauhan NR, Conwell DL, Clancy TE, Banks PA, Silverman SG. Necrotizing pancreatitis: diagnosis, imaging, and intervention. Radiographics. 2014 Sep-Oct; 34 (5): 1218-39. doi: 10. 1148/rg. 345130012 .

14. Lankisch PG, Struckmann K, Assmus C, Lehnick D, Maisonneuve P, Lowenfels AB ScandDo we need a computed tomography examination in all patients with acute pancreatitis within $72 \mathrm{~h}$ after admission to hospital for the detection of pancreatic necrosis? J Gastroenterol. 2001 Apr; 36(4):432-6.

15. Wiesner W, Studler U, Kocher T, Degen L, Buitrago-Tellez $\mathrm{CH}$, Steinbrich W. Colonic involvement in non-necrotizing acute pancreatitis: correlation of CT findings with the clinical course of affected patients. Eur Radiol. 2003 Apr; 13(4): 897-902. Epub 2002 Jun 28.

16. Chamisa I, Mokoena T, Luvhengo TE. Changing Pattern of Incidence, Aaetiology and Mortality from Acute Pancreatitis at Kalafong Hospital, Pretoria, South Africa, 1988-2007: A Retrospective Evaluation. East and Central African Journal of Surgery. 2010;15(1):35-39.

17. Mortelé KJ, Mergo PJ, Taylor HM, Ernst MD, Ros PR. Renal and perirenal space involvement in acute pancreatitis: spiral CT findings. Abdom Imaging. 2000 May-Jun;25(3):272-8.

18. Lecesne R, Taourel P, Bret PM, Atri M, Reinhold C. Acute pancreatitis: interobserver agreement and correlation of CT and MR cholangiopancreatography with outcome. Radiology. 1999 Jun;211(3):727-35.

19. Sharmaa V, Surinder S. Ranaa A study of radiological scoring system evaluating extrapancreatic inflammation with conventional radiological and clinical scores in predicting outcomes in acute pancreatitis Ann Gastroenterol 2015; 28 (1): 1-6www.annalsgastro.gr

20. Chenyang Chen, MD, Zixing HuangEvaluation of extrapancreatic inflammation on abdominal computed tomography as an early predictor of organ failure in acute pancreatitis as defined by the revised Atlanta classification Medicine (Baltimore). 2017 Apr; 96(15): e6517. Published online 2017 Apr 14.

\section{How to cite this article?}

Parida K, Biswal D. Acute Pancreatitis-A Prospective study of Estimation of Prognosis with MCTSI versus CTSI. Int J Med Res Rev 2017;5(06):547-553. doi:10.17511/ijmrr. 2017.i06.02. 